\title{
Spin polarization of electrons induced by strong collisional magnetic fields
}

\author{
Gerhard Soff \\ Gesellschaft für Schwerionenforschung, Darmstadt, West Germany \\ Joachim Reinhardt and Walter Greiner \\ Institut für Theoretische Physik, Johann Wolfgang Goethe Universität, Frankfurt am Main, West Germany
}

(Received 17 March 1980)

\begin{abstract}
We calculate the spin polarization of $1 s \sigma$ vacancies and emitted $\delta$ electrons induced by the strong magnetic field $\left(\left|B_{\max }\right| \sim 10^{16} \mathrm{G}\right)$ in collisions of very heavy ions $\left(Z_{1}+Z_{2}=178\right)$. The electron excitations are determined by the solution of coupled-channel equations within the quasimolecular basis states including the vector potential. The formulation is extended to the many-electron case. Spin polarizations of the order of 5-10\% for impact energies below the Coulomb barrier are predicted.
\end{abstract}

\section{INTRODUCTION}

During the last years more and more interest has focused on the behavior of electrons moving in strong magnetic fields. Several years ago $\mathrm{J}$. Rafelski and B. Müller pointed out ${ }^{1}$ that the strongest magnetic fields on a microscopic scale accessible to experimental observations are created in collisions of very heavy ions with $\left(Z_{1}+Z_{2}\right) \alpha>1$. Their magnitude can simply be estimated by considering the magnetic field strength produced by a circular current along the symmetry axis. It follows from Biot-Savart's law that

$$
|\overrightarrow{\mathrm{B}}(r)|=\frac{1}{2} \mu_{0} \frac{I R^{2}}{\left(r^{2}+R^{2}\right)^{3 / 2}},
$$

where $R$ denotes the radius of the current loop and $r$ the distance from its center. The heavy ion current $I$ is given by $I=\left(Z_{1}+Z_{2}\right) e v_{\text {ion }} / 2 \pi R$. Assuming $Z_{1}+Z_{2}=178, v_{1 \text { on }}=0.1 c$, and $R=15 \mathrm{fm}$, the maximum magnetic field strength $(r=0)$ gets $\left|\overrightarrow{\mathrm{B}}_{\max }\right|=4 \times 10^{15} \mathrm{G}$. In superheavy quasimolecules the $1 s \sigma$ electron moves almost adiabatically close to the nuclei and is highly localized. Hence it may serve as test particle for the high $-\vec{B}$ limit of quantumelectrodynamics. For $r \gg R$, $\overrightarrow{\mathrm{B}}$ is of the dipole-type $|\overrightarrow{\mathrm{B}}(r)| \sim r^{-3}$. The $1 s \sigma^{-}$ Bohr orbit is located at about $r \sim 100 \mathrm{fm}$ where we still find $|\overrightarrow{\mathrm{B}}(r=100 \mathrm{fm})|=10^{13} \mathrm{G}$.

The electric and magnetic field strength as well as the corresponding density distributions for two colliding heavy ions moving on straight lines with classical impact parameter $b$ can be found in Ref. 2. In preceding papers, the Zeeman splitting ${ }^{1}$ of innermost bound electrons and spin polarizations ${ }^{3}$ due to dynamical excitations in superheavy quasimolecules have been calculated using first-order perturbation theory. In this paper we present a treatment of the dynamical behavior of electron configurations under the action of time-varying Coulomb and magnetic fields. Within the adiabatic, quasimolecular picture we solve for the first time the coupledchannel equations for the electron-occupation amplitudes with inclusion of the scalar and vector potentials in the transverse Coulomb gauge. Retardation and electron-screening effects are neglected in our calculations. The first is justified as long as the heavy ions are not relativistic. Electron screening will probably only change the magnitude of ionization, but should have very small effect on the polarization. With an extended version of the developed computer code, in principle, it is possible to predict ionization probabilities and $\delta$-electron distributions for the next heavy ion accelerator generation up to $E_{\mathrm{ion}}=500$ $\mathrm{MeV} / \mathrm{u}$. Different techniques ${ }^{4-6}$ must be applied for the theoretical description of ionization phenomena in relativistic heavy ion collision.

This paper is organized as follows. The singleelectron-coupled channel equations for adiabatic basis states are presented in the next section. In Sec. III the vector potential in the Coulomb as well as in the Lorentz gauge is derived and a multipole expansion is performed. Section IV is devoted to the calculation of the basis states for electron wave functions and to the evaluation of matrix elements of the magnetic interaction. In Sec. $\mathrm{V}$ the many-electron formalism is discussed and expressions for the polarizations of particles and holes are derived. Our numerical results for the system $Z_{1}+Z_{2}=178(\mathrm{~Pb}+\mathrm{Cm})$ are exhibited in Sec. VI. We have chosen this particular quasimolecular system because currently it is the heaviest system where the $1 s \sigma$ ionization probability has already been measured ${ }^{7}$ at GSI.

\section{DYNAMICS OF A SINGLE ELECTRON IN THE FIELD OF TWO COLLIDING HEAVY IONS}

In this chapter we present the basic formalism for single-electron exeitations in collisions of 
very heavy ions. Starting from an adiabatic basis set we calculate the occupation amplitudes $a_{i j}(t)$ of an electron moving in the combined electric and magnetic field of both scattering ions. Our considerations are based on the notations and results of Refs. 8 and 9. The dynamical behavior of the electron $\Phi_{i}$ is described by the time-dependent Schrödinger equation $(\hbar=c=1)$

$$
i \frac{\partial \Phi_{i}}{\partial t}=H \Phi_{i}
$$

where $H$ is the Hamiltonian, which is specified below.

The time-dependent wave function $\Phi_{i}(t)$ is expanded in terms of the complete adiabatic basis states $\phi_{j}$

$\Phi_{i}(\overrightarrow{\mathrm{r}}, t)=\sum_{j=1}^{\infty} a_{i j}(t) \phi_{j}(\overrightarrow{\mathrm{r}}, R(t)) \exp \left[-i \chi_{j}(t)\right]$,

with the phase

$$
\chi_{j}(t)=\int^{t} E_{j}\left(t^{\prime}\right) d t^{\prime} \text {. }
$$

The sum in Eq. (2.2) is understood to include an integration over continuum states. The basis states $\phi_{f}$ are determined as solutions of the stationary two-center Dirac equation ${ }^{1,10,11}$

$$
\begin{aligned}
H_{0} \phi_{j} & =\left[\vec{\alpha} \overrightarrow{\mathrm{p}}+\beta m_{e}+V_{\mathrm{TC}}(\overrightarrow{\mathrm{r}}, R(t))\right] \phi_{j}(\overrightarrow{\mathrm{r}}, R(t)) \\
& =E_{j}(R(t)) \phi_{j}(\overrightarrow{\mathrm{r}}, R(t)) .
\end{aligned}
$$

The method of solution first proposed in Ref. 1 has been extensively discussed in Ref. 12. The relativistic Dirac Hamiltonian $\mathrm{H}_{0}$ depends on time parametrically via the internuclear separation $R(t)$ entering in the two-center Coulomb potential $V_{\mathrm{TC}}(\overrightarrow{\mathrm{r}}, R(t))$.

The total Hamiltonian in (2.1) is split into

$$
H=H_{0}+H^{\prime},
$$

where $H^{\prime}$ contains any interaction responsible for electron excitations which is not included in $H_{0}$. In the case of magnetic interactions it is given by

$$
H^{\prime}=-\vec{\alpha} \cdot \overrightarrow{\mathrm{A}}
$$

with the vector potential $\vec{A}$ created by the current of both colliding ions. A factor $-e$ is included in the definition of the potentials $V$ and $\vec{A}$. Inserting the ansatz (2.2) into Eq. (2.1) followed by projection leads to a set of first order coupled dif- ferential equations for the occupation amplitudes $a_{i j}(t)$,

$$
\begin{gathered}
\dot{a}_{i j}(t)=-\sum_{k=1}^{\infty} a_{i k}(t)\left(\left\langle\phi_{j}\left|\frac{\partial}{\partial t}\right| \phi_{k}\right\rangle+i\left\langle\phi_{j}\left|H^{\prime}(t)\right| \phi_{k}\right\rangle\right) \\
\times \exp \left(\dot{i}_{\chi_{j k}}\right),
\end{gathered}
$$

with the phases

$$
\chi_{j k}=\chi_{j}-\chi_{k} \text {. }
$$

As usual, we split the $\partial / \partial t$ operator into its radial and rotational coupling components

$$
\frac{\partial}{\partial t}=\dot{R} \frac{\partial}{\partial R}-i \vec{\omega} \cdot \vec{J} \text {. }
$$

The computation of the $\partial / \partial t$ coupling matrix element is described in detail in Ref. 12. We adopt the same notation and the results of that article.

\section{THE VECTOR POTENTIAL OF TWO COLLIDING NUCLEI}

Starting from elementary classical electrodynamics we first will derive the vector potential in the Coulomb gauge created by the colliding nuclei. Except for a gauge function the scalar potential $V$ and the vector potential $\vec{A}$ are determined by the solutions of the inhomogenous wave equations $^{13}(c=1)$ :

$$
\begin{aligned}
& \nabla^{2} V+\frac{\partial}{\partial t}(\vec{\nabla} \cdot \overrightarrow{\mathrm{A}})=4 \pi \rho e, \\
& \nabla^{2} \overrightarrow{\mathrm{A}}-\frac{\partial^{2}}{\partial t^{2}} \overrightarrow{\mathrm{A}}-\vec{\nabla}\left(\vec{\nabla} \cdot \overrightarrow{\mathrm{A}}+\frac{\partial V}{\partial t}\right)=4 \pi \overrightarrow{\mathrm{J}} e,
\end{aligned}
$$

where $\rho$ and $\vec{J}$ denote the heavy ion charge and current density, respectively. Assuming pointlike nuclei we have

$$
\begin{aligned}
& \rho(\overrightarrow{\mathrm{r}}, t)=\sum_{i=1,2} Z_{i} e \delta\left(\overrightarrow{\mathrm{r}}-\overrightarrow{\mathrm{R}}_{i}(t)\right), \\
& \overrightarrow{\mathrm{J}}(\overrightarrow{\mathrm{r}}, t)=\sum_{i=1,2} Z_{i} e \overrightarrow{\mathrm{v}}_{i}(t) \delta\left(\overrightarrow{\mathrm{r}}-\overrightarrow{\mathrm{R}}_{i}(t)\right),
\end{aligned}
$$

with $\overrightarrow{\mathrm{v}}_{i}$ being the heavy ion velocity. The transverse Coulomb gauge is defined by

$$
\vec{\nabla} \cdot \vec{A}_{c}=0
$$

and hence one obtains in this gauge the instantaneous Coulomb potential as

$$
V_{c}(\overrightarrow{\mathbf{r}}, t)=-e \int \frac{\rho\left(\overrightarrow{\mathbf{r}}^{\prime}, t\right)}{\left|\overrightarrow{\mathbf{r}}^{\prime}-\overrightarrow{\mathbf{r}}^{\prime}\right|} d^{3} r^{\prime}
$$

With Eq. (3.3) the two-center Coulomb potential becomes

$$
V_{c}(\overrightarrow{\mathrm{r}}, t)=-\frac{Z_{1} e^{2}}{\left|\overrightarrow{\mathrm{r}}-\overrightarrow{\mathrm{R}}_{1}(t)\right|}-\frac{Z_{2} e^{2}}{\left|\overrightarrow{\mathrm{r}}-\overrightarrow{\mathrm{R}}_{2}(t)\right|} .
$$


The vector potential obeys the differential equation

$$
\nabla^{2} \overrightarrow{\mathrm{A}}_{c}-\frac{\partial^{2}}{\partial t^{2}} \overrightarrow{\mathrm{A}}_{c}=e 4 \pi \overrightarrow{\mathrm{J}}+\vec{\nabla} \frac{\partial V_{c}}{\partial t}=4 \pi e \overrightarrow{\mathrm{J}}_{t},
$$

with the transverse current. ${ }^{13}$

$$
\overrightarrow{\mathrm{J}}_{t}(\overrightarrow{\mathrm{r}}, t)=\frac{1}{4 \pi} \vec{\nabla} \times \vec{\nabla} \times \int \frac{\overrightarrow{\mathrm{J}}\left(\overrightarrow{\mathbf{r}}^{\prime}, t\right)}{\left|\overrightarrow{\mathbf{r}}-\overrightarrow{\mathrm{r}}^{\prime}\right|} d^{3} r^{\prime} .
$$

\section{Writing}

$$
\vec{J}_{t}=\vec{J}-\vec{J}_{t}
$$

and with the longitudinal current

$$
\overrightarrow{\mathrm{J}}_{l}(\overrightarrow{\mathrm{r}}, t)=-\frac{1}{4 \pi} \vec{\nabla} \int \frac{\vec{\nabla} \cdot \cdot \overrightarrow{\mathrm{J}}\left(\overrightarrow{\mathrm{r}}^{\prime}, t\right)}{\left|\overrightarrow{\mathbf{r}}-\overrightarrow{\mathrm{r}}^{\prime}\right|} d^{3} r^{\prime},
$$

one finds with Eq. $(3.4)^{13}$

$$
\begin{aligned}
\overrightarrow{\mathrm{J}}_{t}(\overrightarrow{\mathrm{r}}, t)=\sum_{i=1,2} Z_{i} e & {\left[\frac{-1}{4 \pi} \vec{\nabla}\left(\overrightarrow{\mathrm{v}}_{i}(t) \cdot \frac{\left[\overrightarrow{\mathrm{r}}-\overrightarrow{\mathrm{R}}_{i}(t)\right]}{\left|\overrightarrow{\mathrm{r}}-\overrightarrow{\mathrm{R}}_{i}(t)\right|^{3}}\right)\right.} \\
& \left.+\overrightarrow{\mathrm{v}}_{i}(t) \delta\left(\overrightarrow{\mathrm{r}}-\overrightarrow{\mathrm{R}}_{i}(t)\right)\right] .
\end{aligned}
$$

In the Coulomb gauge (3.5) all magnetic and retardation effects are completely contained in the vector potential. On the other hand, the Lorentz gauge

$$
\vec{\nabla} \cdot \vec{A}_{L}+\frac{\partial V_{L}}{\partial t}=0
$$

leads to the equations

$$
\nabla^{2} V_{L}-\frac{\partial^{2}}{\partial t^{2}} V_{L}=4 \pi \rho e
$$

and

$$
\nabla^{2} \overrightarrow{\mathrm{A}}_{L}-\frac{\partial^{2}}{\partial t^{2}} \overrightarrow{\mathrm{A}}_{L}=4 \pi \overrightarrow{\mathrm{J}} e
$$

Employing the retarded propagator

$$
G\left(\overrightarrow{\mathrm{r}}, t ; \overrightarrow{\mathrm{r}}^{\prime}, t^{\prime}\right)=\frac{\delta\left(t^{\prime}-t+\left|\overrightarrow{\mathrm{r}}-\overrightarrow{\mathrm{r}}^{\prime}\right|\right)}{\left|\overrightarrow{\mathrm{r}}-\overrightarrow{\mathrm{r}}^{\prime}\right|},
$$

the solutions can be expressed as

$$
\begin{aligned}
& V_{L}(\overrightarrow{\mathrm{r}}, t)=-e \int \frac{\rho\left(\overrightarrow{\mathrm{r}}^{\prime}, t-\left|\overrightarrow{\mathrm{r}}-\overrightarrow{\mathrm{r}}^{\prime}\right|\right)}{\left|\overrightarrow{\mathrm{r}}-\overrightarrow{\mathrm{r}}^{\prime}\right|} d^{3} r^{\prime}, \\
& \overrightarrow{\mathrm{A}}_{L}(\overrightarrow{\mathrm{r}}, t)=-e \int \frac{\overrightarrow{\mathrm{J}}\left(\overrightarrow{\mathrm{r}}, t-\left|\overrightarrow{\mathrm{r}}-\overrightarrow{\mathrm{r}}^{\prime}\right|\right)}{\left|\overrightarrow{\mathrm{r}}-\overrightarrow{\mathrm{r}}^{\prime}\right|} d^{3} r^{\prime} .
\end{aligned}
$$

In the same way it results from Eq. (3.8)

$$
\overline{\mathrm{A}}_{c}(\overrightarrow{\mathrm{r}}, t)=-e \int \frac{\overrightarrow{\mathrm{J}}_{t}\left(\overrightarrow{\mathrm{r}}, t-\left|\overrightarrow{\mathrm{r}}-\overrightarrow{\mathrm{r}}^{\prime}\right|\right)}{\left|\overrightarrow{\mathrm{r}}-\overrightarrow{\mathrm{r}}^{\prime}\right|} d^{\beta} r^{\prime} .
$$

As a basic approximation we neglect now all retardation effects $\left(t-\left|\overrightarrow{\mathbf{r}}-\overrightarrow{\mathbf{r}}^{\prime}\right| \sim t\right)$. Their influence on the scalar potential was estimated ${ }^{14}$ to be of the order of $0.1 \%$ for heavy ion velocities below the Coulomb barrier. Thus we employ the mag- netostatic expression

$$
\overline{\mathrm{A}}_{c}(\overrightarrow{\mathrm{r}}, t)=-e \int \frac{\overrightarrow{\mathrm{J}}_{t}\left(\overrightarrow{\mathrm{r}}^{\prime}, t\right)}{\left|\overrightarrow{\mathrm{r}}-\overrightarrow{\mathrm{r}}^{\prime}\right|} d^{3} r^{\prime} .
$$

Inserting now the transverse current (3.12) and integrating by parts finally yields for the vector potential in the Coulomb gauge ${ }^{13}$

$$
\begin{aligned}
\overrightarrow{\mathrm{A}}_{c}(\overrightarrow{\mathrm{r}}, t)= & -\sum_{i=1,2} \frac{Z_{i} e^{2} \overrightarrow{\mathrm{v}}_{i}(t)}{2\left|\overrightarrow{\mathrm{r}}-\overline{\mathrm{R}}_{i}(t)\right|} \\
& -\sum_{i=1,2} \frac{Z_{i} e^{2} \overrightarrow{\mathrm{v}}_{i}(t) \cdot\left[\overrightarrow{\mathrm{r}}-\overrightarrow{\mathrm{R}}_{i}(t)\right]\left[\overrightarrow{\mathrm{r}}-\overrightarrow{\mathrm{R}}_{i}(t)\right]}{2\left|\overrightarrow{\mathrm{r}}-\overrightarrow{\mathrm{R}}_{i}(t)\right|^{3}} \\
= & \vec{A}_{c}^{(1)}+A_{c}^{(2)} .
\end{aligned}
$$

The corresponding result in the Lorentz-gauge, $\overrightarrow{\mathrm{A}}_{\mathbf{L}}(\overrightarrow{\mathrm{r}}, t)$ follows from (3.18), (3.4), and (3.21) neglecting also retardation:

$$
\overline{\mathrm{A}}_{L}(\overline{\mathrm{r}}, t)=2 A_{c}^{(1)}(\overline{\mathrm{r}}, t)
$$

We investigate now the influence of the vector potential (3.21) in the Coulomb gauge on spin polarizations of $K$ vacancies and $\delta$ electrons. This problem has been already studied inconsistently in Ref. 3 employing time-dependent perturbation theory for the dynamical excitations of electrons. The inconsistency came about with the choice of the quasimolecular basis states, calculated with the scalar potential (3.7) in the Coulomb gauge, while the vector potential was treated in the Lorentz gauge (3.22). However; we shall see that according to the correct results of this article, the final conclusions of Ref. 3 remain valid.

For simplicity we will restrict our following derivations to symmetric systems with

$$
Z_{1}=Z_{2}=Z
$$

and choose our coordinate system according to

$$
\begin{aligned}
& \vec{R}_{1}=-\vec{R}_{2}=-\frac{1}{2} \vec{R}, \\
& \vec{v}_{1}=-\vec{v}_{2}=-\frac{1}{2} \vec{v},
\end{aligned}
$$

i.e., the coordinates are measured from the geometrical center of the colliding system. The nuclear trajectories are prescribed as Rutherford hyperbolas in the $x-z$ plane with the $z$ axis connecting both nuclei. $\vec{R}(t)$ and $\vec{v}(t)$ denote the internuclear separation and the relative velocity of both nuclei. $\vec{v}$ can be divided into its radial and azimuthal components

$$
\overrightarrow{\mathrm{v}}=v_{R} \overrightarrow{\mathrm{e}}_{R}+v_{\phi} \overrightarrow{\mathrm{e}}_{\phi}=v_{R} \overrightarrow{\mathrm{e}}_{z}+v_{\phi} \overrightarrow{\mathrm{e}}_{x},
$$

with the radial velocity $v_{R}=\dot{R}(t)$ and the rotational 
velocity

$$
v_{\phi}=\frac{v_{\infty} b}{R(t)}
$$

$R=|\overrightarrow{\mathrm{R}}|, v_{\infty}=|\overrightarrow{\mathrm{v}}(t=\infty)|$ is the asymptotic heavy ion velocity and $b$ denotes the classical impact parameter.

Next we perform a multipole expansion of the vector potential (3.21). For that purpose we shall make use of the following expansions ${ }^{15}$ :

$$
\frac{1}{|\overrightarrow{\mathrm{r}}+\overrightarrow{\mathrm{a}}|}=\sum_{l=0}^{\infty}( \pm 1)^{l} \frac{r_{l}^{l}}{r_{>}^{l+1}} P_{l}(\cos \gamma)
$$

with $\boldsymbol{r}_{<}=\min (|\overrightarrow{\mathbf{r}}|,|\overrightarrow{\mathrm{a}}|)$, and $\boldsymbol{r}_{>}=\max (|\overrightarrow{\mathbf{r}}|,|\overrightarrow{\mathrm{a}}|)$. Also

$$
\begin{aligned}
& \frac{r^{2}-a^{2}}{|\overrightarrow{\mathrm{r}} \mp \overrightarrow{\mathrm{a}}|^{3}}=-\sum_{l=0}^{\infty}( \pm 1)^{l}(2 l+1) \frac{r^{l}}{a^{l+1}} P_{l}(\cos \gamma), \\
& \frac{a \mp r \cos \gamma}{|\overrightarrow{\mathrm{r}} \mp \overrightarrow{\mathrm{a}}|^{3}}=\sum_{l=0}^{\infty}( \pm 1)^{l}(l+1) \frac{r^{l}}{a^{l+2}} P_{l}(\cos \gamma),
\end{aligned}
$$

for $r<a$; and

$$
\begin{aligned}
& \frac{r^{2}-a^{2}}{|\overrightarrow{\mathrm{r}} \mp \overrightarrow{\mathrm{a}}|}=\sum_{l=0}^{\infty}( \pm 1)^{l}(2 l+1) \frac{a^{l}}{r^{l+1}} P_{l}(\cos \gamma), \\
& \frac{ \pm a-r \cos \gamma}{|\overrightarrow{\mathrm{r}} \mp \overrightarrow{\mathrm{a}}|^{3}}=\mp \sum_{l=1}^{\infty}( \pm 1)^{l} l \frac{a^{l-1}}{r^{l+1}} P_{l}(\cos \gamma) .
\end{aligned}
$$

for $r>a$. With (3.28) we immediately derive for the Lorentz term in $(3.21)\left(\overrightarrow{\mathrm{a}}=\frac{1}{2} \overrightarrow{\mathrm{R}}\right)$

$$
\begin{aligned}
\overrightarrow{\mathrm{A}}_{c}^{(1)} & =\frac{1}{2} \overrightarrow{\mathrm{A}}_{L}=-\left(v_{R} \overrightarrow{\mathrm{e}}_{z}+v_{\phi} \overrightarrow{\mathrm{e}}_{x}\right) \frac{Z e^{2}}{2} \sum_{l=1,3}^{\infty} \frac{r_{l}^{l}}{r_{>}^{l+1}} P_{l}(\cos \gamma) \\
& =-\frac{1}{2} Z e^{2}\left(G_{z} \overrightarrow{\mathrm{e}}_{z}+G_{x} \overrightarrow{\mathrm{e}}_{x}\right)
\end{aligned}
$$

where $\gamma$ denotes the polar angle with respect to the $z$ axis. The multipole expansion of the gauge $\operatorname{term} A_{c}^{(2)}$ is more complicated. According to our choice of the coordinate system we can write

$$
\begin{aligned}
& \overrightarrow{\mathrm{A}}_{c}^{(2)}=-\frac{1}{4} Z e^{2}(-\left[v_{\phi} x+v_{R}\left(z+\frac{1}{2} R\right)\right] \frac{\overrightarrow{\mathbf{r}}+\frac{1}{2} \overrightarrow{\mathbf{R}}}{\left|\overrightarrow{\mathbf{r}}+\frac{1}{2} \overrightarrow{\mathbf{R}}\right|^{3}} \\
&\left.+\left[v_{\phi} x+v_{R}\left(z-\frac{1}{2} R\right)\right] \frac{\overrightarrow{\mathbf{r}}-\frac{1}{2} \overrightarrow{\mathbf{R}}}{\left|\overrightarrow{\mathbf{r}}-\frac{1}{2} \overrightarrow{\mathbf{R}}\right|^{3}}\right)
\end{aligned}
$$

and we may decompose this gauge term into

$$
\overrightarrow{\mathrm{A}}_{c}^{(2)}=-\frac{1}{2} Z e^{2}\left(F_{r} \overrightarrow{\mathrm{e}}_{r}+F_{z} \overrightarrow{\mathrm{e}}_{z}\right) \text {. }
$$

Notice that $\vec{e}_{r}$ and $\vec{e}_{z}$ are not orthogonal. With $z=r \cos \gamma$ and the expansion (3.29)-(3.32), it follows that

$$
\begin{aligned}
F_{r}=\{\mp\} v_{R} \frac{r}{\frac{1}{2} R} \sum_{l=0,2}^{\infty}\left\{\begin{array}{c}
i+1 \\
l
\end{array}\right\} P_{l}(\cos \gamma) \frac{r_{l}^{l}}{r_{>}^{l+1}} \\
+v_{\phi} \frac{1}{r_{>}^{2}-r_{<}^{2}} \frac{r}{\frac{1}{2} R} \sum_{l=1,3}^{\infty}(2 l+1) \frac{x}{r} P_{l}(\cos \gamma) \frac{r_{l}^{l+1}}{r_{>}^{l}}
\end{aligned}
$$

$$
\begin{aligned}
F_{z}=\{ \pm\} v_{R} \sum_{l=1,3}^{\infty}\left\{\begin{array}{c}
l+1 \\
l
\end{array}\right\} P_{l}(\cos \gamma) \frac{r_{l}^{l}}{r_{>}^{l+1}} \\
-v_{\phi} \frac{1}{r_{>}^{2}-r_{<}^{2}} \sum_{l=0,2}^{\infty}(2 l+1) \frac{x}{r} P_{l}(\cos \gamma) \frac{r_{l}^{l+1}}{r_{>}^{l}}
\end{aligned}
$$

In (3.36) and (3.37) the upper (lower) line is valid for $r<\frac{1}{2} R\left(r>\frac{1}{2} R\right)$. The fictitious divergence in the $v_{\phi}$-terms can be eliminated by combining the corresponding succeeding terms of the series expansions. Finally one can make use of the relation

$$
\frac{x}{r} P_{l}(\cos \gamma)=\sqrt{2 \pi} \sum_{l^{\prime}}\left(2 l^{\prime}+1\right)^{1 / 2}\left[\begin{array}{lll}
1 & l & l^{\prime} \\
1 & 0 & -1
\end{array}\right]\left[\begin{array}{lll}
1 & l & l^{\prime} \\
0 & 0 & 0
\end{array}\right]\left[Y_{l^{\prime}, 1}(\cos \gamma)-Y_{l^{\prime},-1}(\cos \gamma)\right]
$$

Now the vector potential induced by the heavy ion current is expressed by the radial coordinate $r$ and the polar angle $\gamma$. If equation (3.38) is inserted into (3.36) one finds after some elementary transformations

$$
F_{r}=\left\{\mp v_{R} \frac{r}{\frac{1}{2} R} \sum_{l=0,2}^{\infty}\left\{\begin{array}{c}
l+1 \\
l
\end{array}\right\} P_{l}(\cos \gamma) \frac{r_{l}^{l}}{r_{>}^{l+1}}-v_{\phi} \frac{r}{\frac{1}{2} R} \sum_{l=1,3}^{\infty} \sqrt{2 \pi}\left(\frac{(l+1)(l+2)}{2(2 l+3)}\right)^{1 / 2}\left[Y_{l+1,1}(\cos \gamma)-Y_{l+l,-1}(\cos \gamma)\right] \frac{r_{l}^{l+1}}{r_{>}^{l+2}}\right. \text {. }
$$

In the same way one obtains for $F_{z}$

$$
F_{z}=\{ \pm\} v_{R} \sum_{l=1,3}^{\infty}\left\{\begin{array}{c}
l+1 \\
l
\end{array}\right\} P_{l}(\cos \gamma) \frac{r_{l}^{l}}{r_{>}^{l+1}}+v_{\phi} \sum_{l=0,2}^{\infty} \sqrt{2 \pi}\left(\frac{(l+1)(l+2)}{2(2 l+3)}\right)^{1 / 2}\left[Y_{l+1,1}(\cos \gamma)-Y_{l+l,-1}(\cos \gamma)\right] \frac{r_{+}^{l+1}}{r_{>}^{l+2}} .
$$




\section{ELECTRONIC WAVEFUNCTIONS AND MAGNETIC COUPLING MATRIX ELEMENTS}

In order to solve the stationary Dirac equation (2.4) with the two-center potential (3.7) and (3.24) in the Coulomb gauge, we perform a multipole expansion $^{1,12}$ of the basis state wavefunction $\phi(\overrightarrow{\mathrm{r}})$ :

$$
\phi_{\mu}(\overrightarrow{\mathrm{r}})=\sum_{\kappa= \pm 1}^{ \pm \infty}\left(\begin{array}{ll}
g_{k}(r) & \chi_{k}^{\mu} \\
i f_{k}(r) & \chi_{i_{k}}^{\mu}
\end{array}\right),
$$

with $^{16}$

$$
\chi_{\kappa}^{\mu}=\sum_{m= \pm 1 / 2}\left(l, \frac{1}{2}, j, \mu-m, m\right) Y_{l}^{\mu-m}(\gamma) \chi^{m} .
$$

$f_{k}(r)$ and $g_{k}(r)$ are small and large components of the radial wavefunction. $\kappa$ is connected to the angular momentum through

$$
\kappa=\left\{\begin{array}{l}
l \text { for } j=l-\frac{1}{2}, \\
-l-1 \text { for } j=l+\frac{1}{2},
\end{array}\right.
$$

with the total angular momentum $j=|\kappa|-\frac{1}{2}$. The magnetic quantum number $\mu$ denotes the projec- tion of the total angular momentum on the axis connecting the two nuclei ( $z$ axis). With (3.28) also the two-center Coulomb potential can be expanded into multipoles which yield for symmetric systems and pointlike nuclei

$$
V(\overrightarrow{\mathrm{r}}, R)=\sum_{i=0,2}^{\infty} V_{l}(r, R) P_{l}(\cos \gamma),
$$

with $\left(Z_{1}=Z_{2}=Z\right)$

$$
V_{l}(r, R)=-2 Z e^{2} \frac{r_{l}^{l}}{r_{>}^{l+l}} \text {. }
$$

The coupled radial equations then read (see, e.g., Ref. 12)

$$
\begin{aligned}
\frac{d}{d r} g_{k}(r)= & (E+m) f_{k}(r)-\frac{\kappa+1}{r} g_{k}(r) \\
& -\sum_{R_{1} l} f_{\mathrm{k}}(r) V_{l}(r, R) A_{(-\kappa, l,-R)}^{\mu}, \\
\frac{d}{d r} f_{k}(r)= & \frac{\kappa-1}{r} f_{k}(r)-(E-m) g_{k}(r) \\
& +\sum_{k_{l} l} g_{k}(r) V_{l}(r, R) A_{(\kappa, l, R)}^{\mu},
\end{aligned}
$$

For the numerical treatment of eqs. (4.6) it is convenient to transform the radial differential equations to a logarithmic scale. Writing $F_{k}$ $=r f_{k}, G_{k}=r g_{k}$, and $r=r_{0} e^{x}$, it follows that

$$
\begin{aligned}
& \frac{d G_{\kappa}}{d x}=-\kappa G_{\kappa}+(E+m) F_{\kappa} r-\sum_{k, l} F_{k} V_{l} r A_{(-\kappa, l,-k)}^{\mu}, \\
& \frac{d F_{K}}{d x}=\kappa F_{\kappa}-(E-m) G_{\kappa} r+\sum_{k_{0} l} G_{\bar{k}} V_{l} r A_{(\kappa, l, k)}^{\mu} .
\end{aligned}
$$

Extensive numerical studies of the two-center Dirac equation for electronic bound states were performed by Betz et al. for the symmetric systems $\mathrm{Pb}+\mathrm{Pb}$ and $\mathrm{U}+\mathrm{U}$ in Refs. 12 and 17 and for the asymmetric system $\mathrm{Pb}+\mathrm{Cm}$ in Ref. 18 . With the multipole expansion of the vector potential (Sec. III) and the two center wavefunctions we are now able to evaluate matrix elements of the magnetic interaction for bound states

$$
M=\left\langle\phi_{\mu^{\prime}}\left|H^{\prime}\right| \phi_{\mu}\right\rangle \text {. }
$$

Using Eqs. (2.6), (3.33), (3.39), and (3.40) one can write

$$
H^{\prime}=\frac{1}{2} Z e^{2}\left[\left(G_{z}+F_{z}\right) \alpha_{z}+G_{x} \alpha_{x}+F_{r} \alpha_{r}\right] .
$$

$$
\left[\left(2 l_{\kappa}+1\right)\left(2 l_{R}+1\right)\right]^{1 / 2}\left[\begin{array}{lll}
l_{\kappa} & l & l_{R} \\
0 & 0 & 0
\end{array}\right]\left[\begin{array}{lll}
l_{\kappa} & l & l_{\mathbb{R}} \\
m-\mu & 0 & \mu-m
\end{array}\right]
$$

$\vec{\alpha}$ is expressed in the standard representation ${ }^{16}$ by

$$
\vec{\alpha}=\left[\begin{array}{ll}
0 & \vec{\sigma} \\
\vec{\sigma} & 0
\end{array}\right]
$$

The angular integration in $(4.9)$ can be performed analytically.

In heavy ion collisions excitations to the continuum play a major role for inner-shell vacancy formation $^{8,9}$ and can therefore not be neglected. However, continuum solutions of the two-center Dirac equation are unknown at present. Therefore we are forced to apply the monopole approximation of the two-center potential, where only the term with $l=0$ in the expansion (4.4) is retained. This monopole approximation was first proposed in Refs. 19-21. Its validity has been investigated in Refs. 12 and 22. Then the basis states simplify to wavefunctions with good angular momentum ${ }^{16}$ :

$$
\phi_{k \mu}(\vec{r})=\left[\begin{array}{ll}
g(r) & \chi_{k}^{\mu} \\
i f(r) & \chi_{-\kappa}^{\mu}
\end{array}\right],
$$


with the usual radical equations

$$
\begin{aligned}
& \frac{d g}{d r}=\left(E+m-V_{0}\right) f-\frac{\kappa+1}{r} g, \\
& \frac{d f}{d r}=\frac{\kappa-1}{r} f-\left(E-m-V_{0}\right) g .
\end{aligned}
$$

The monopole potential for pointlike nuclei is simply ${ }^{12}$

$V_{0}(r, R)= \begin{cases}-\frac{4 Z e^{2}}{R} \text { for } r<\frac{1}{2} R, \\ -\frac{2 Z e^{2}}{r} \text { for } r>\frac{1}{2} R .\end{cases}$

For the more realistic case of extended nuclei we refer to the discussions of Refs. 12, 22, and 23. Equations $(4.13)$ have been solved numerical-

$1 y^{12}$ for both bound and continuum states. For the calculation of excitations induced by the vector potential and of the diagonal interactions leading to a Zeeman splitting of electron states we still need the matrix elements (4.9).

First we consider matrix elements of the type

$$
M_{r}=\left\langle\phi_{\kappa^{\prime} \mu^{\prime}}^{\prime}\left|D_{L}(r) \alpha_{r} Y_{L M}\right| \phi_{\kappa \mu}\right\rangle,
$$

with $L$ being even and $M=-1,0,+1$. Using

$$
\alpha_{r}=\left(\begin{array}{cc}
0 & \sigma_{r} \\
\sigma_{r} & 0
\end{array}\right)
$$

and $d^{16}$

$$
\sigma_{\tau} \chi_{\kappa}^{\mu}=-\chi_{-\kappa}^{\mu},
$$

we find after angular integration ${ }^{24,25}$

$$
M_{r}=-i \int_{0}^{\infty} r^{2} d r D_{L}(r)\left[g^{\prime} f X\left(l^{\prime}, j^{\prime}, \mu^{\prime} ; l, j, \mu ; L, M\right)-f^{\prime} g X\left(\bar{l}^{\prime}, j^{\prime}, \mu^{\prime} ; \bar{l}, j, \mu ; L, M\right)\right],
$$

with the abbreviation

$$
\begin{aligned}
X\left(l^{\prime}, j^{\prime}, \mu^{\prime} ; l, j, \mu, ; L, M\right)=\sum_{m= \pm 1 / 2}(-1)^{\mu^{\prime}-m}\left(l^{\prime}, \frac{1}{2}, j^{\prime}, \mu^{\prime}-m, m\right)\left(l, \frac{1}{2}, j, \mu-m, m\right) & \\
& \times\left(\frac{\left(2 l^{\prime}+1\right)(2 l+1)(2 L+1)}{4 \pi}\right)^{1 / 2}\left(\begin{array}{lll}
l^{\prime} & l & L \\
-\mu^{\prime}+m & \mu-m & M
\end{array}\right]\left[\begin{array}{lll}
l^{\prime} & l & L \\
0 & 0 & 0
\end{array}\right) .
\end{aligned}
$$

Here $l$ and $j$ denote the orbital and total angular momentum related to $\kappa$, whereas $\bar{l}$ and $j$ are connecter: with $-\kappa$ and can be taken from Eq. (4.3). The selection rules for this matrix element are $\mu^{\prime}=\mu+M$ and $l^{\prime}+l+L$ being even.

Next we consider the matrix elements

$$
M_{z}=\left\langle\phi_{\kappa^{\prime} \mu^{\prime}}^{\prime}\left|D_{L}(r) \alpha_{z} Y_{L M}\right| \phi_{\kappa \mu}\right\rangle,
$$

with

$$
\sigma_{z}=\left[\begin{array}{ll}
1 & 0 \\
0 & -1
\end{array}\right] \text {. }
$$

Here $L$ is odd and again $M=-1,0,+1$. Thus we get

$$
M_{z}=i \int_{0}^{\infty} r^{2} d r D_{L}(r)\left[g^{\prime} f Y\left(l^{\prime}, j^{\prime}, \mu^{\prime} ; \bar{l}, j, \mu ; L, M\right)-f^{\prime} g Y\left(\vec{l}, j^{\prime}, \mu^{\prime} ; l, j, \mu ; L, M\right],\right.
$$

with the abbreviation

$$
\begin{aligned}
Y\left(l^{\prime}, j^{\prime}, \mu^{\prime} ; l, j, \mu ; L, M\right)=\sum_{m= \pm 1 / 2} & \operatorname{sgn} m(-1)^{\mu^{\prime}-m}\left(l^{\prime}, \frac{1}{2}, j^{\prime}, \mu^{\prime}-m, m\right)\left(l, \frac{1}{2}, j, \mu-m, m\right) \\
& \times\left(\frac{\left(2 l^{\prime}+1\right)(2 l+1)(2 L+1)}{4 \pi}\right)^{1 / 2}\left(\begin{array}{lll}
l^{\prime} & l & L \\
-\mu^{\prime}+m & \mu-m & M
\end{array}\right]\left(\begin{array}{lll}
l^{\prime} & l & L \\
0 & 0 & 0
\end{array}\right) .
\end{aligned}
$$

$M_{z}$ differs from $M_{r}$ only by the signum in the sum and by the exchange of the angular momentum quantum numbers $\bar{l}$ and $l$. This leads to the modified selection rule: $l^{\prime}+\bar{l}+L$ is even. 
Finally it is necessary to calculate the matrix elements

$$
M_{x}=\left\langle\phi_{\kappa^{\prime} \mu^{\prime}}\left|D_{L}(r) \alpha_{x} Y_{L O}\right| \phi_{\kappa \mu}\right\rangle
$$

with

$$
\sigma_{x}=\left[\begin{array}{ll}
0 & 1 \\
1 & 0
\end{array}\right) .
$$

After angular integration one obtains ( $L$ is odd)

$$
M_{x}=i \int_{0}^{\infty} r^{2} d r D_{l}(r)\left[g^{\prime} f Z\left(l^{\prime}, j^{\prime}, \mu^{\prime} ; \bar{l}, j, \mu ; L, 0\right)-f^{\prime} g Z\left(\bar{l}^{\prime}, j^{\prime}, \mu^{\prime} ; l, j, \mu ; L, 0\right)\right] \text {, }
$$

with

$$
\begin{aligned}
Z\left(l^{\prime}, j^{\prime}, \mu^{\prime} ; l, j, \mu ; L, 0\right)=\sum_{m= \pm 1 / 2}(-1)^{\mu^{\circ}+m}\left(l^{\prime}, \frac{1}{2}, j^{\prime}, \mu^{\prime}+m,-m\right)\left(l, \frac{1}{2}, j, \mu-m, m\right) \\
\quad \times\left(\frac{\left(2 l^{\prime}+1\right)(2 l+1)(2 L+1)}{4 \pi}\right)^{1 / 2}\left[\begin{array}{lll}
l^{\prime} & l & L \\
-\mu^{\prime}-m & \mu-m & 0
\end{array}\right]\left(\begin{array}{lll}
l^{\prime} & l & L \\
0 & 0 & 0
\end{array}\right) .
\end{aligned}
$$

Obviously this matrix element exists only between states with $\mu^{\prime}=\mu \pm 1 . l^{\prime}+\bar{l}+L$ must be even.

Having obtained all results relevant for the general case now we focus our attention to the magnetic coupling between $n s \sigma$ states in the monopole approximation $\left(j=\frac{1}{2}, l=0, \bar{l}=1\right)$. Then the angular momentum coefficients simplify to

$$
\begin{aligned}
& X\left(0, \frac{1}{2}, \pm \frac{1}{2} ; 0, \frac{1}{2}, \pm \frac{1}{2} ; 0,0\right)=X\left(1, \frac{1}{2}, \pm \frac{1}{2} ; 1, \frac{1}{2}, \pm \frac{1}{2} ; 0,0\right)=\frac{1}{\sqrt{4 \pi}}, \\
& Y\left(0, \frac{1}{2}, \pm \frac{1}{2} ; 1, \frac{1}{2}, \pm \frac{1}{2} ; 1,0\right)=Y\left(1, \frac{1}{2}, \pm \frac{1}{2} ; 0, \frac{1}{2}, \pm \frac{1}{2} ; 1,0\right)=-\frac{1}{\sqrt{3}} \frac{1}{\sqrt{4 \pi}}, \\
& Y\left(0, \frac{1}{2}, \mp \frac{1}{2} ; 1, \frac{1}{2}, \pm \frac{1}{2} ; 1, \mp 1\right)=-Y\left(1, \frac{1}{2}, \mp \frac{1}{2} ; 0, \frac{1}{2}, \pm \frac{1}{2} ; 1, \mp 1\right)= \pm\left(\frac{2}{3}\right)^{1 / 2} \frac{1}{\sqrt{4 \pi}}, \\
& Z\left(0, \frac{1}{2}, \mp \frac{1}{2} ; 1, \frac{1}{2}, \pm \frac{1}{2} ; 1,0\right)=-Z\left(1, \frac{1}{2}, \mp \frac{1}{2} ; 0, \frac{1}{2}, \pm \frac{1}{2} ; 1,0\right)=\mp \frac{1}{\sqrt{3}} \frac{1}{\sqrt{4 \pi}} .
\end{aligned}
$$

'All other possible coupling vanish due to the selection rules or due to the triangle rule. If we insert the values $(4.28)-(4.31)$ into the corresponding matrix elements we find that all contributions to couplings between $s$ states with the same magnetic quantum number just cancel in the Coulomb gauge:

$$
\left\langle s^{\prime} \pm \frac{1}{2}\left|H_{\text {Coulomb }}^{\prime}\right| s \pm \frac{1}{2}\right\rangle=0 .
$$

This is different from the Lorentz gauge, where we obtain

$$
\begin{aligned}
& \left\langle s^{\prime} \pm \frac{1}{2}\left|H_{\text {Lorentz }}^{\prime}\right| s \pm \frac{1}{2}\right\rangle \\
& \quad=-\frac{Z e^{2}}{3} i v_{R} \int_{0}^{\infty} r^{2} d r \frac{r_{L}}{r_{>}^{2}}\left(f g^{\prime}-f^{\prime} g\right) .
\end{aligned}
$$

However, also in this case there is no contribution to the Zeeman splitting: Due to the minus sign in the integrand of (4.33) the diagonal matrix elements $\left(s^{\prime}=s\right)$ vanish. For the spin-flip transitions we find in summary the simple result

$$
\begin{aligned}
& \left\langle s^{\prime} \mp \frac{1}{2}\left|H^{\prime}\right| s \mp \frac{1}{2}\right\rangle \\
& \quad=\mp \frac{Z e^{2}}{3} i v_{\Phi} \int_{0}^{\infty} r^{2} d r \frac{r_{<}}{r_{>}^{2}}\left(f g^{\prime}+f^{\prime} g\right) \\
& \quad=\mp i A_{s^{\prime} \neq, s \pm},
\end{aligned}
$$

which is the same in the Coulomb as well as in the Lorentz gauge. Futhermore we note that according to the selection rules in symmetric systems, all magnetic mixed couplings between $s \pm \frac{1}{2}$ and $p_{1 / 2} \pm \frac{1}{2}$ states vanish in the monopole approximation.

For the magnetic coupling between $p_{1 / 2}$ states we found the same results as for the $s_{1 / 2}$ states except for an overall minus sign in Eq. (4.34). In just the same way as we treated the magnetic interaction it is possible to investigate the influence of higher electric multipoles $(l \geqslant 1)$ of Eq. (4.4) on $n s \sigma$ states in the monopole approximation. In this case we have to compute the matrix elements

$$
M_{e}=\left\langle\phi_{\kappa^{\prime} \mu^{\prime}}^{\prime}\left|H_{e}\right| \phi_{\kappa \mu}\right\rangle \text {, }
$$

with

$$
H_{e}=\sum_{i=1}^{\infty} V_{t} P_{l}(\cos \gamma)
$$

Using the angular integrals (4.7) we find immediately that $M_{e}=0$ for states with $\kappa=-1$, which again underlines the validity of the monopole approximation for the calculation of $n s \sigma$ ionization. The radial coupling matrix element of Eq. 
(2.7) and (2.9) are presented in Ref. 12. The only nonvanishing matrix elements of rotational interaction involving $s$ states in the monopole approximation are determined by $^{25}$

$$
\left\langle s \pm \frac{1}{2}\left|J_{y}\right| s \mp \frac{1}{2}\right\rangle=\mp \frac{1}{2} i
$$

with

$$
\omega_{y}=b v_{\infty} / R^{2}
$$

this leads to

$$
\left\langle s \pm \frac{1}{2}|-i \vec{\omega} \vec{J}| s \mp \frac{1}{2}\right\rangle=\mp b v_{\infty} / 2 R^{2} .
$$

\section{POLARIZATIONS AND MANY-ELECTRON ASPECTS}

Before we start to discuss explicitly polarization phenomena, we first derive some symmetry relations for the occupation amplitudes. For this purpose we introduce the abbreviation

$$
D_{j, k+}=D_{j-, k-}=D_{j, k}=\left\langle\phi_{j \pm}\left|\dot{R} \frac{\partial}{\partial R}\right| \phi_{k \pm}\right\rangle \text {, }
$$

where the "+" and "_-" sign denotes the corresponding spin projection. The matrix elements of magnetic interaction (4.34) obey the following relations:

$$
A_{j-, k+}=-A_{j+, k-}=-A_{k+j_{-}}=A_{k-, j+}=A_{j, k} .
$$

The coupled channel equations (2.7) then read

$$
\begin{aligned}
\dot{a}_{i+, j+}= & -\sum_{r+} a_{i+, r+} D_{j r} e^{-i x_{r j}} \\
& +\sum_{r-} a_{i+, r-} A_{j r} e^{-i x_{r j}}, \\
\dot{a}_{i-, j-}= & -\sum_{r-} a_{i-, r-} D_{j r} e^{-i x_{r j}} \\
& -\sum_{r+} a_{i-, r+} A_{j r} e^{-i x_{r j}},
\end{aligned}
$$

and

$$
\begin{aligned}
\dot{a}_{i+, j-}= & -\sum_{r-} a_{i+, r-} D_{j r} e^{-i x_{r j}}+\sum_{r+} a_{i+, r+} A_{j r} e^{-i x_{r j}}, \\
\dot{a}_{i-, j+}= & -\sum_{r+} a_{i-, r+} D_{j r} e^{-i x_{r j}} \\
& +\sum_{r-} a_{i-, r-} A_{j r} e^{-i x_{r j}}
\end{aligned}
$$

which have to be solved with the initial conditions

$$
a_{i+, j+}(t=-\infty)=\delta_{i+, j+}
$$

or

$$
a_{i_{-, j-}}(t=-\infty)=\delta_{i-, j-_{-}} .
$$

If we neglect the magnetic interaction, the spinflip amplitudes (5.5) and (5.6) will vanish.

The two sets of differential equations are solved consistently if the following symmetry relations hold:

$$
\begin{aligned}
\text { and } & a_{i+, r+}=a_{i-, r-} \\
a_{i+, r-} & =-a_{i-, r_{+}} .
\end{aligned}
$$

These results imply, that no spin polarization with respect to the quantization axis (the $z$ axis connecting both nuclei) can be observed. The polarization is expected to be oriented in the direction of the magnetic field strength, i.e., the $y$ axis or thogonal to the scattering plane. In order to calculate the spin polarizations along the $y$ axis we have to rotate the coordinate system applying the rotational matrixes $D_{m m}^{j}(\alpha, \beta, \gamma)^{24}$ :

$$
D_{m m}^{j}(\alpha, \beta, \gamma)=e^{-i \alpha m^{\prime}} d_{m m}^{j}(\beta) e^{-i \gamma m},
$$

with

$$
\begin{aligned}
& d_{1 / 2,1 / 2}^{1 / 2}=d_{-1 / 2,-1 / 2}^{1 / 2}=\cos \left(\frac{1}{2} \beta\right), \\
& d_{-1 / 2,1 / 2}^{1 / 2}=-d_{1 / 2,-1 / 2}^{1 / 2}=\sin \left(\frac{1}{2} \beta\right) .
\end{aligned}
$$

For this purpose we represent the wavefunction by

$$
\Phi=c_{z} \chi_{z}^{1 / 2}+c_{z-} \chi_{z}^{-1 / 2}
$$

This leads to

$$
\begin{aligned}
& \chi_{z}^{1 / 2}=D_{1 / 2,1 / 2}^{1 / 2} \chi_{y}^{1 / 2}+D_{-1 / 2,1 / 2}^{1 / 2} \chi_{y}^{-1 / 2}, \\
& \chi_{z}^{-1 / 2}=D_{1 / 2,-1 / 2}^{1 / 2} \chi_{y}^{1 / 2}+D_{-1 / 2,-1 / 2}^{1 / 2} \chi_{y}^{-1 / 2},
\end{aligned}
$$

and correspondingly for the amplitudes

$$
\begin{aligned}
& c_{y_{+}}=D_{1 / 2,1 / 2}^{1 / 2} c_{z_{+}}+D_{1 / 2,-1 / 2}^{1 / 2} c_{z_{-}}, \\
& c_{y_{-}}=D_{-1 / 2,1 / 2}^{1 / 2} c_{z_{+}}+D_{-1 / 2,-1 / 2}^{1 / 2} c_{z_{-}} .
\end{aligned}
$$

Inserting now the Euler angles $\alpha=\frac{3}{2} \pi, \beta=\frac{1}{2} \pi$, $\gamma=\frac{1}{2} \pi$ we finally get for the amplitudes with respect to the spin projection along the $y$ axis

$$
\begin{aligned}
& c_{y_{+}}=\frac{1}{\sqrt{2}}\left(-c_{z+}+i c_{z-}\right), \\
& c_{y-}=\frac{1}{\sqrt{2}}\left(i c_{z+}-c_{z^{-}}\right) .
\end{aligned}
$$

So far we have described only the fate of a single electron influenced by the collision dynamics. Now we start to discuss some aspects of the many electron problem ${ }^{8,9,26-29}$ where we follow the lines pointed out in Ref. 8. We expand the field operator $\hat{\psi}$ in terms of the complete adiabatic basis states $\phi_{\mathbf{a}}$ :

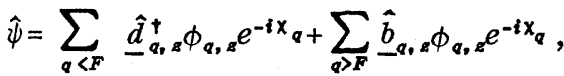

$$
\begin{aligned}
& =\sum_{a<F} \underline{\hat{d}}_{a, y}^{+} \phi_{a, y} e^{-i \chi_{a}}+\sum_{q>F} \hat{\hat{b}}_{a, y} \phi_{a, y} e^{-i \chi_{a}},
\end{aligned}
$$

where the subscript " $z$ " or " $y$ " denotes quantiza- 
tion along the $z$ or $y$ axis, respectively. From

(5.17) we find the operator relation $(q<F)$

$$
\underline{\hat{d}}_{a, y}^{\dagger}=\sum_{a<F}\left\langle\phi_{a, y} \mid \phi_{a, z}\right\rangle \underline{\hat{d}}_{a, z}^{\dagger},
$$

with

$$
\begin{aligned}
& \left\langle\phi_{a+, y} \mid \phi_{a+, z}\right\rangle=\left\langle\phi_{a-, y} \mid \phi_{a-, z}\right\rangle=-1 / \sqrt{2}, \\
& \left\langle\phi_{a+, y} \mid \phi_{a-, z}\right\rangle=\left\langle\phi_{a-, y} \mid \phi_{a+, z}\right\rangle=i / \sqrt{2} .
\end{aligned}
$$

The number of created holes $q$ with positive-spin projection $(q+<F)$ along the $y$ axis therefore is

$$
\begin{aligned}
& N_{\alpha+}^{y}=\left\langle\psi\left|\underline{\hat{d}}_{\alpha+, y}^{+} \underline{\hat{d}}_{\alpha+, y}\right| \psi\right\rangle \\
& =\frac{1}{2}\left\langle\psi\left|\underline{\hat{d}}_{q+, z}^{\dagger} \underline{\hat{d}}_{a+z}\right| \psi\right\rangle+\frac{1}{2}\left\langle\psi\left|\underline{\hat{d}}_{q^{-}, z}^{\dagger} \underline{\hat{d}}_{q-, z}\right| \psi\right\rangle \\
& +\frac{1}{2} i\left\langle\psi\left|\underline{\hat{d}}_{q+, \Sigma}^{\dagger} \underline{\hat{d}}_{\alpha-, \Sigma}\right| \psi\right\rangle-\frac{1}{2} i\left\langle\psi\left|\underline{\hat{d}}_{q-, \Sigma}^{\dagger} \underline{\hat{d}}_{q+, z}\right| \psi\right\rangle .
\end{aligned}
$$

Alternatively, we may expand $\hat{\psi}$ in terms of the single-particle wave functions $\Phi_{q}(t)$ with definite boundary condition at $t \rightarrow-\infty$, already containing the dynamical excitations

$$
\hat{\psi}=\sum_{q<F} \hat{d}_{q}^{\dagger} \Phi_{q}(t)+\sum_{q>F} \hat{b}_{q} \Phi_{q}(t)
$$

with the definitions for operators for holes

$$
\hat{d}_{q}=\hat{b}_{q}^{\dagger} \text { for } q<F
$$

and

$$
\hat{d}_{q}^{\dagger}=\hat{b}_{q} \text { for } q<F .
$$

Comparing (5.22) and (5.17) we are left with

$$
\begin{aligned}
& \hat{\tilde{d}}_{a \pm, z}^{\dagger}=\sum_{r+<F} a_{r+, \alpha \pm} \hat{d}_{r+}^{+}+\sum_{r=<F} a_{r-, \alpha \pm} \hat{d}_{r-}^{+} \\
& +\sum_{s+>F} a_{s+, q \pm} \hat{b}_{s+}+\sum_{s \rightarrow \boldsymbol{F}} a_{s-, q \pm} \hat{b}_{s-}
\end{aligned}
$$

This transformation may be employed to evaluate the required expectation values of Eq. (5.21) since the operators $\hat{d}_{q}, \underline{\hat{b}}_{q}$ are constructed such as to destroy the Heisenberg ground-state vector $|\psi\rangle$, i.e.,

$$
\begin{aligned}
& \hat{b}_{s \pm}|\psi\rangle=0 \text { for } s>F, \\
& \hat{d}_{r \pm}|\psi\rangle=0 \text { for } r<F,
\end{aligned}
$$

and

$$
\left[\hat{b}_{i \pm}, \hat{b}_{j \pm}^{\dagger}\right]=\delta_{i \pm, j \pm} .
$$

All other anticommutators vanish. In particular, the additional expectation values of the type

$$
M_{a}=\left\langle\psi\left|\hat{d}_{q \pm, z}^{\dagger} \hat{d}_{q \mp, z}\right| \psi\right\rangle
$$

which did not appear in Ref. 8 lead to

$$
M_{a}=\sum_{s \downarrow F} a_{s+, q \mp}^{*} a_{s+, q \pm}+\sum_{s \rightarrow>F} a_{s-, q \mp}^{*} a_{s-, q \pm}
$$

Finally we obtain for the number of created holes $N_{q}^{y}$ with spin projection along the $y$ axis

$$
\begin{aligned}
N_{q \pm}^{y}= & \frac{1}{2}\left(\sum_{s+>F}\left|a_{s+q q}\right|^{2}+\sum_{s=>F}\left|a_{s-, q+}\right|^{2}\right)+\frac{1}{2}\left(\sum_{s+>F}\left|a_{s+, q-}\right|^{2}+\sum_{s->F}\left|a_{s-, q-}\right|^{2}\right) \\
& -\frac{i}{2}\left(\sum_{s+>F} a_{s+, q \pm}^{*} a_{s+, q F}+\sum_{s \rightarrow>F} a_{s-, q \pm}^{*} a_{s-, q F}\right)+\frac{i}{2}\left(\sum_{s+>F} a_{s+, q F}^{*} a_{s+, q \pm}+\sum_{s=>F} a_{s-, q F}^{*} a_{s-, q \pm}\right) .
\end{aligned}
$$

The spin polarization of holes $q$ along the $y$ axis is defined by

$$
\eta_{q}=\frac{N_{q+}^{y_{+}}-N_{q_{-}}^{y}}{N_{q+}^{y}+N_{q-}^{y}}
$$

This yields

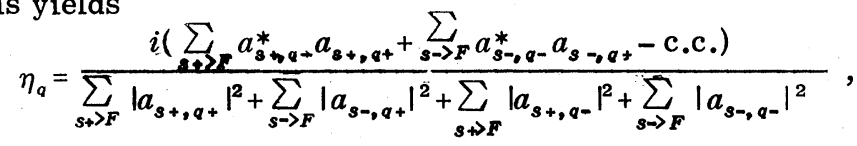

where c.c. denotes the complex conjugate. The same structure as in (5.34) can be obtained simply by taking the $\sigma_{y}$ expectation value of $\Phi_{a}$. With

$$
\Phi_{q}=c_{+} \chi_{z}^{1 / 2}+c_{-} \chi_{z}^{-1 / 2}
$$

and

$$
\sigma_{y}=\left(\begin{array}{cc}
0 & -i \\
i & 0
\end{array}\right)
$$


it follows that

$$
\eta_{q}^{\prime}=\frac{\left\langle\Phi_{q}\left|\sigma_{q}\right| \Phi_{q}\right\rangle}{\left\langle\Phi_{q} \mid \Phi_{q}\right\rangle}=-i \frac{\left(c_{+}^{*} c_{-}-c_{-}^{*} c_{+}\right)}{\left|c_{+}\right|^{2}+\left|c_{-}\right|^{2}},
$$

which supports the validity of our result (5.33). With the completeness relation ${ }^{8}$

$$
\sum_{i} a_{k i}^{*} a_{i i}=\sum_{i} a_{i k}^{*} a_{i l}=\delta_{k l},
$$

the time-reversal-symmetry relation

$$
\left|a_{s q}(t=\infty)\right|=\left|a_{q s}(t=\infty)\right|,
$$

and the symmetry relations (5.9) and (5.10), one can rewrite $\eta_{q}$ and simplify Eq. (5.33).

$$
\eta_{q}=\frac{2 \operatorname{Im}\left(\sum_{r<F} a_{q-, r+}^{*} a_{q-, r-}\right)}{1-\sum_{r=<F}\left|a_{q-, r-}\right|^{2}-\sum_{r+<F}\left|a_{q-, r+}\right|^{2}}
$$

In just the same manner we can also evaluate the number of created particles $p$ (e.g., $\delta$-electrons) with spin polarizations along the $y$-axis.

$$
\left.N_{p}^{y}=\left\langle\psi\left|\underline{\hat{b}}_{p, y}^{\dagger} \underline{\hat{b}}_{p, y}\right| \psi\right\rangle \text { for } p\right\rangle F .
$$

We obtain

$$
N_{p+}^{y}=\frac{1}{2}\left\langle\psi\left|\underline{\hat{b}}_{p+, z}^{\dagger} \underline{\hat{b}}_{p+, z}\right| \psi\right\rangle+\frac{1}{2}\left\langle\psi\left|\underline{\hat{b}}_{p-, z}^{\dagger} \underline{\hat{b}}_{p-, z}\right| \psi\right\rangle+\frac{1}{2} i\left\langle\psi\left|\underline{\hat{b}}_{p-, z}^{\dagger} \underline{\hat{b}}_{p+, z}\right| \psi\right\rangle-\frac{i}{2}\left\langle\psi\left|\underline{\hat{b}}_{p+, z}^{\dagger} \underline{\hat{b}}_{p-, z}\right| \psi\right\rangle
$$

With

$$
\hat{b}_{p \pm, z}=\sum_{r+<F} a_{r+, p \pm} \hat{d}_{r+}^{\dagger}+\sum_{r=<F} a_{r-, p \pm} \hat{d}_{r-}^{\dagger}+\sum_{s+>F} a_{s+, p \pm} \hat{b}_{s+}+\sum_{s->F} a_{s-, p \pm} \hat{b}_{s-},
$$

it follows for the matrix elements of the type

$$
M_{b}=\left\langle\psi\left|\underline{b}_{p \pm, z}^{\dagger} \hat{b}_{p \mp, z}\right| \psi\right\rangle=\sum_{r+\langle F} a_{r+, p \pm}^{*} a_{r+, p F}+\sum_{r<<F} a_{r-, p \pm}^{*} a_{r-, p F} .
$$

The number of created particles then yields

$$
\begin{aligned}
N_{p \pm}^{y}= & \frac{1}{2}\left(\sum_{r+<F}\left|a_{r+, p+}\right|^{2}+\sum_{r=<F}\left|a_{r-, p+}\right|^{2}\right)+\frac{1}{2}\left(\sum_{r+<F}\left|a_{r+, p-}\right|^{2}+\sum_{r<F}\left|a_{r-, p-p}\right|^{2}\right) \\
& +\frac{1}{2} i\left(\sum_{r+<F} a_{r+, p *}^{*} a_{r+, p *}+\sum_{r=<F} a_{r-, p \neq}^{*} a_{r-, p \pm}\right)-\frac{1}{2} i\left(\sum_{r+<F} a_{r+, p \pm}^{*} a_{r+, p F}+\sum_{r-<F} a_{r-, p \pm}^{*} a_{r-, p *}\right) .
\end{aligned}
$$

The spin polarization of particles along the $y$ axis is

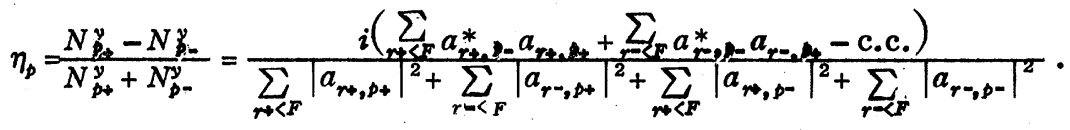

Again this may be rewritten as

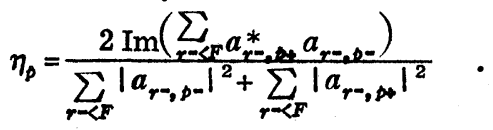

Furthermore we note the fact that particles and holes have opposite spin polarizations. Finally we remark that the spin polarization along the $x$ axis vanishes as was expected on physical grounds.

VI. RESULTS AND DISCUSSION

We solved the coupled-channel equations (5.3)(5.6) for the $n s \sigma$ states by numerical integration. In the monopole approximation, the radial coupling
(5.1), the rotational coupling (4.38), and the diagonal and off-diagonal magnetic interaction (4.34) are taken into account. Since in the present computations spin-up and spin-down states have to 


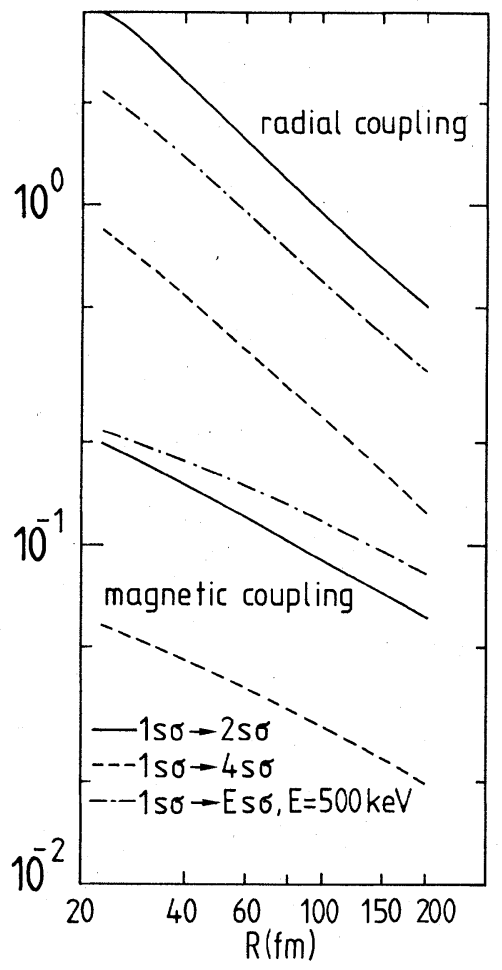

FIG. 1. Comparison of the radial and magnetic coupling strength in units of $\hbar=m_{e}=c=1$ versus internuclear separation $R$. We show $\left|D_{1 s \sigma, n s \sigma} / \dot{R}\right|[$ [Eq. (5.1)] and sep$\left|A_{1 s-\sigma, n s+\sigma} / v_{\phi}\right|$ [Eq. (4.34)] for $n=2,4$ and a continuum state with kinetic energy $E=500 \mathrm{keV}$, respectively $\left(Z_{1}+Z_{2}=178\right)$.

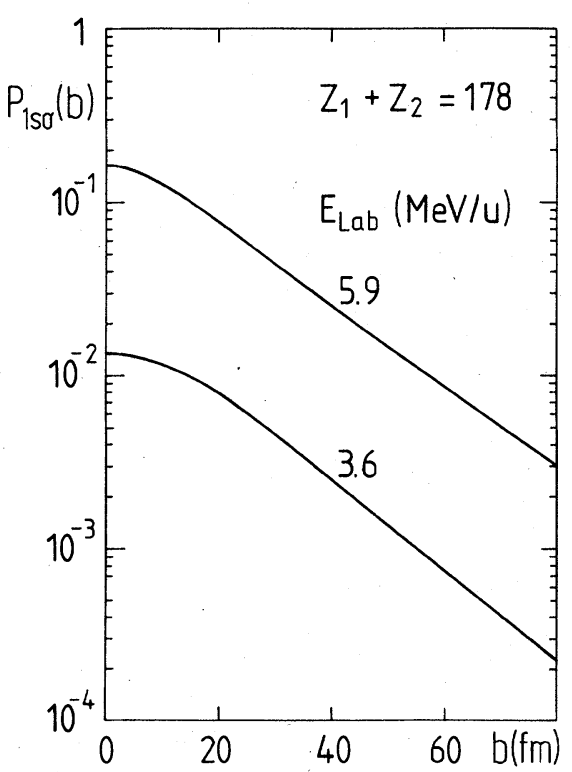

FIG. 2. Number of created $1 s \sigma$ vacancies per collision with impact parameter $b$. Two different ion energies are considered for the system $Z_{1}+Z_{2}=178$.

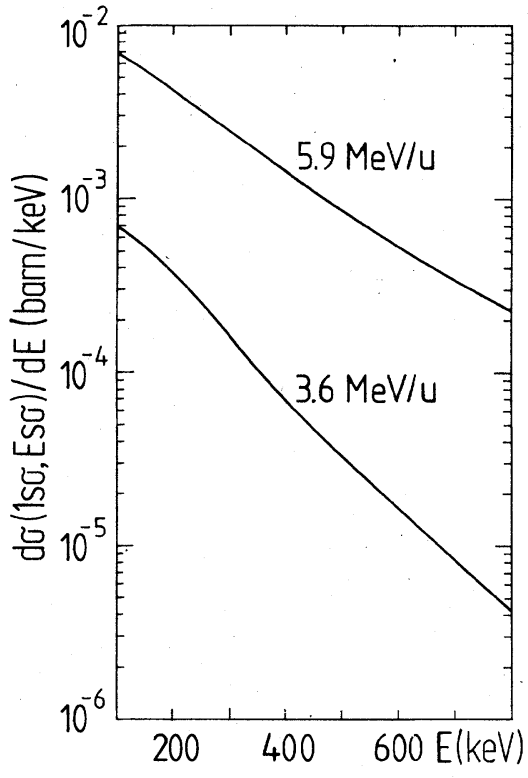

FIG. 3. Differential cross section for $\delta$-electron emission with respect to kinetic electron energy. The number of correlated $\delta$ electrons and $1 s \sigma$ vacancies are considered using Eq. (36) of Ref. 8.

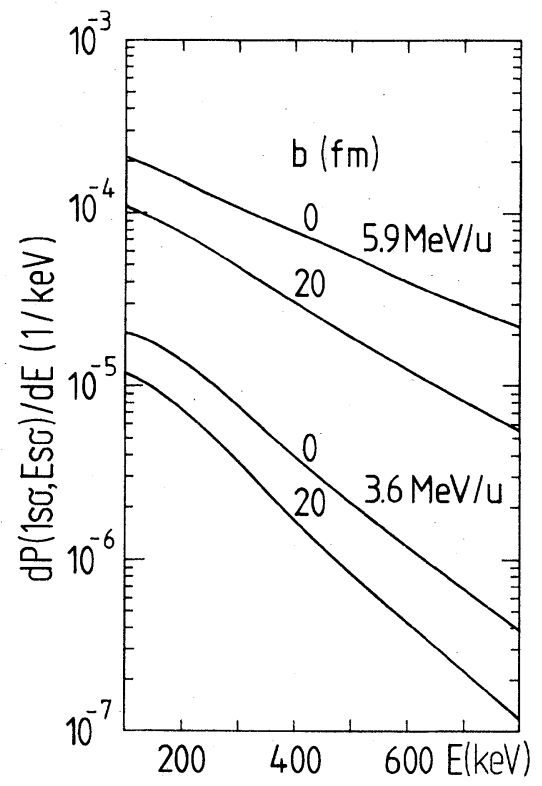

FIG. 4. Differential probability versus kinetic electron energy for emitted $\delta$-electrons correlated with $1 s \sigma$ vacancies at the impact parameters $b=0$ and $20 \mathrm{fm}$. 


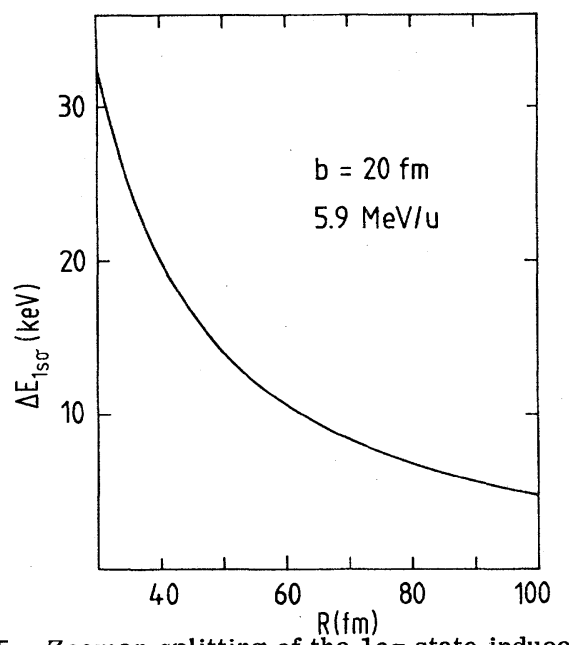

FIG. 5. Zeeman splitting of the $1 s \sigma$ state induced by the strong magnetic field in the colliding system $Z_{1}+Z_{2}$ $=178$.

be treated explicitly we had to reduce the number of considered basis states slightly compared with the calculations of Ref. 8. As bound states we included the $1 s \sigma$ up to the $5 s \sigma$ state whereas the integration over the positive energy continuum between $E=m c^{2}$ and $E=3 m c^{2}$ has been performed with 13 grid points. The dimension of the remaining system of coupled-channel equations therefore is $n=72$ (real and imaginary part treated separately). In Fig. 1 the absolute value of the radial coupling (5.1) $\left|D_{1 s \sigma, n s \sigma} / \dot{R}\right|$ and the magnetic coupling (4.34) $\left|A_{1 s-\sigma, n s+\sigma} / v_{\phi}\right|$ is compared for $n=2,4$ and for a continuum state with kinetic energy $E=500 \mathrm{keV}$. The off-diagonal magnetic coupling strength is found to be typically by an order of magnitude smaller than the radial coupling for the considered two-center distances between $R=23$ and $200 \mathrm{fm}$. One should keep in

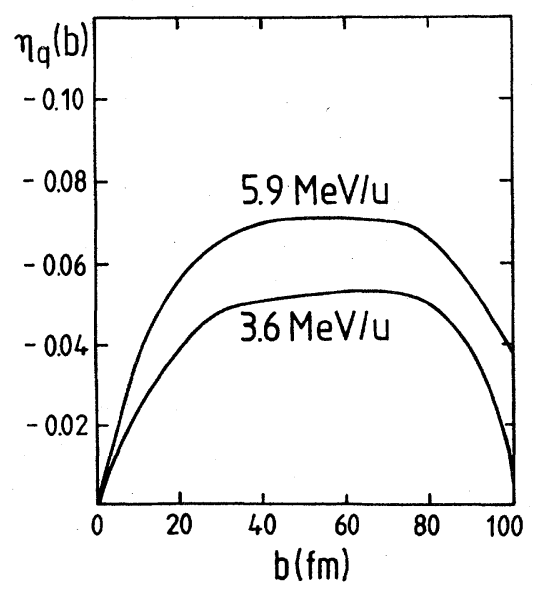

FIG. 6. Spin polarizations of $1 s \sigma$ vacancies versus impact parameter.

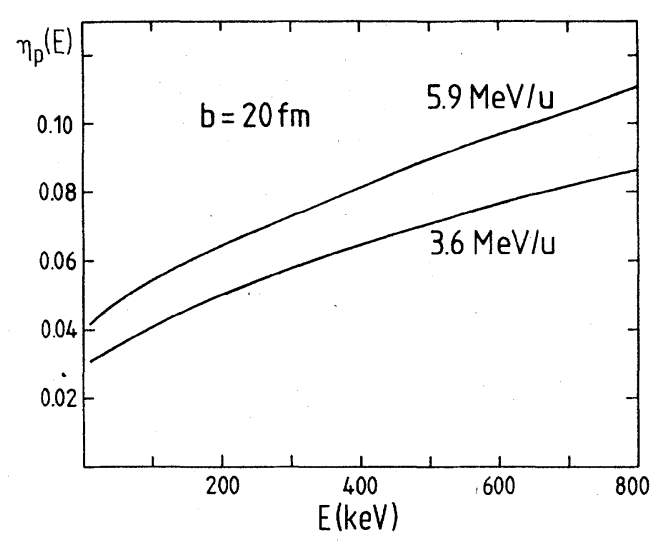

FIG. 7. Spin polarizations of emitted $\delta$ electrons stemming directly from the $1 s \sigma$ state versus kinetic electron energy, using Eq. (5.46).

mind that these matrix elements enter almost quadratically in the excitation probability. Therefore only a minor contribution to the vacancy production probabilities due to the time-varying potential can be expected. As a main result we found that the additional magnetic interaction does not change the total ionization probabilities but leads to spin polarizations of electron states. The created polarization is preserved during the collision despite the importance of the multistep excitation processes. As dominant effect the Zeeman splitting of the $1 s \sigma$ state gives rise to a stronger ionization of one of the usually degenerated spin states whereas the other becomes less ionized. However, the total sum remains almost exactly the same as obtained with the radial coupling only. The number of created $1 s \sigma$ vacancies per collision with impact parameter $b$

$$
P_{1 s o}(b)=2\left(1-\sum_{r<F}\left|a_{1 s o, r}\right|^{2}\right)
$$

is shown in Fig. 2 for the system $Z_{1}+Z_{2}=178$ with $E_{1 \mathrm{ab}}=5.9$ and $3.6 \mathrm{MeV} / u$. The Fermi surface $F,{ }^{8}$ denoting the highest level being occupied at the beginning of the collision $(t=-\infty)$ was chosen to be $F=4 s \sigma$ 。 For central collisions we found $P_{1 s \sigma}(0)=0.16$ and 0.013 , respectively, which is about $10 \%$ smaller compared with the corresponding probabilities of Refs. 20 and 21 due to the reduction of the number of basis states. The $\delta$-electron spectrum for the same system and impact energies is displayed in Fig. 3. A coincidence measurement of $\delta$ electrons with measured $1 s \sigma$ vacancies is required. For the corresponding calculation of the number of correlated particlehole pairs we refer to the formalism of Ref. 8 . The differential probability for $\delta$-electron emission with respect to kinetic electron energy for the impact parameters $b=0$ and $20 \mathrm{fm}$ can be taken 
from Fig. 4. For higher heavy ion energy, the $\delta$-electron distribution shows a weaker dependence on $E$ reflecting the higher Fourier frequencies attainable in this collision.

The Zeeman splitting of the $1 s \sigma$ level as function of internuclear separation $R$ is given in Fig. 5 for a collision with $E_{12 b}=5.9 \mathrm{MeV} / u$ and $b=20$ fm. Using Eq. (4.34) it has been evaluated according to

$$
\Delta E_{1 s \sigma}=2\left|A_{1 s+\sigma, 1 s-\sigma}\right|^{2} .
$$

At the distance of closest approach it reaches a maximum values of $\Delta E(R=30 \mathrm{fm})=32 \mathrm{keV}$.

The spin polarization $\eta_{a}$ of created $1 s \sigma$ vacancies has been calculated according to Eq. (5.40). Its dependence on impact parameter is presented in Fig. $6(q=1 s \sigma)$. As can be seen, $\eta_{q}=0$ for head-on collisions since the rotational velocity $v_{\phi}$ from (3.27) is zero in this case. Its maximum value is obtained for medium impact parameters between $b=30$ and $80 \mathrm{fm}$. For larger impact parameters (distances) the magnetic field is too small in order to influence ionization processes. The calculated spin polarization is of the same order of magnitude as estimated earlier in Ref. 3 within time-dependent perturbation theory. This theoretical prediction still needs experimental verification. The spin polarization $\eta_{p}$ of $\delta$ electrons versus kinetic electron energy $E$ stemming directly from ionization of the $1 s \sigma$ state [Eq. (5.47)] is presented in Fig. 7 for $b=20 \mathrm{fm}$. Correlation effects ${ }^{8}$ are not considered in this case. Larger polarizations are found for increasing $\delta$ electron energy. Ionization measurements of $1 s \sigma$ electrons, therefore, may yield direct information about the behavior of electrons in strong magnetic fields. They may also be of some importance for astrophysical phenomena. In particular astrophysical processes ${ }^{30-34}$ in pulsar magnetospheres and neutron stars as well as electron binding energies ${ }^{35-41}$ of atoms in uniform external magnetic fields have been the subject of widespread theoretical investigations during the last years.

\section{ACKNOWLEDGMENTS}

We are grateful to Prof. Müller for fruitful discussions and for critical reading of the manuscript. This work was supported by the Bundesministerium für Forschung und Technologie and by the Forschungsgemeinschaft (Heisenberg Programm).
${ }^{1}$ J. Rafelski and B. Müller, Phys. Rev, Lett. 36,517 (1976).

${ }^{2}$ G. Soff, W. Betz, J. Kirsch, V. Oberacker, J. Reinhardt, K. -H. Wietschorke, B. Müller, and W. Greiner, Proceedings Predeal International School, Heavy Ion Physics, 1978, Bucharest, Romania, edited by A. Berinde, V. Ceausescu, I. A. Dorobantu, p. 889.

${ }^{3}$ W. Greiner, B. Müller, and G. Soff, Phys. Lett. $69 \mathrm{~A}$, 27 (1978).

${ }^{4}$ R. Anholt, Phys. Rev. A 19, 1004 (1979).

${ }^{5}$ D. M. Davidovic, B. L. Moiseiwitsch, and P.H. Norington, J. Phys. B 11, 847 (1978).

${ }^{6}$ E. Teubner, G. Terlecki, N. Grün, and W. Scheid, J. Phys. B 13, 523 (1980).

${ }^{7}$ D. Liesen, P. Armbruster, F. Bosch, S. Hagmann, P. H. Mokler, H. J. Wollersheim, H. Schmidt-Böcking, R. Schuch, and J. B. Wilhelmy, Phys. Rev. Lett. $\underline{44}$, 983 (1980).

${ }^{8}$ G. Soff, J. Reinhardt, B. Müller, and W. Greiner, Z. Physik A 294, 137 (1980).

${ }^{9} \mathrm{~J}$. Reinhardt, B. Müller, W. Greiner, and G. Soff, Phys. Rev. Lett. 43, 1307 (1979).

${ }^{10}$ B. Müller, J. Rafelski, and W. Greiner, Phys. Lett. 47B, 5 (1973).

${ }^{11}$ B. Müller and W. Greiner, Z. Naturforsch. A31, 1 (1976).

${ }^{12}$ G. Soff, W. Greiner, W. Betz, and B. Müller, Phys. Rev. A 20, 169 (1979).

${ }^{13} \mathrm{~J}$. D. Jackson, Classical Electrodynamics (John Wiley, New York, 1962); W. Greiner, Vorlesungenüber Theoretische Physik III: Elektrodynamik Nerlag Harri
Deutsch, 'Frankfurt a. M., 1978).

${ }^{14}$ D. Kolb, W. -D. Sepp, B. Fricke, and T. Morovic, Z. Physik A 286, 169 (1978).

${ }^{15} \mathrm{G}$. Arfken, Mathematical Methods for Physicists (Academic, New York, 1970).

${ }^{16}$ M. E. Rose, Relativistic Electron Theory (Wiley, New York, 1961).

${ }^{17}$ W. Betz, G. Heiligenthal, B. Müller, V. Oberacker, J. Reinhardt, W. Schäfer, G. Soff, and W. Greiner, Proceeding Predeal International Summer School, 1976, edited by V. Ceausescu, I. A. Dorobantu, (Bucharest, 1977), p. 1.

${ }^{18} \mathrm{~J}$. Reinhardt, W. Betz, J. Kirsch, B. Müller, W. Greiner, and G. Soff, Nukleonika 25, 225 (1980).

${ }^{19}$ B. Müller, J. Rafelski, and W. Greiner, Z. Phys. 257, 183 (1972).

${ }^{20} \mathrm{~J}$. Rafelski, B. Müller, and W. Greiner, Lett. Nuovo Cim. 4, 469 (1972).

${ }^{21}$ V. S. Popov, Yad. Fiz. 17, 621 (1973) [Sov. J. Nucl. 17,322 (1973)].

${ }^{22} \bar{G}$. Soff, J. Reinhardt, W. Betz, and J. Rafelski, Phys. Scr. 17, 417 (1978).

${ }^{23}$ K. -H. Wietschorke, B. Müller, W. Greiner, and G. Soff, J. Phys. B 12, L31 (1979).

${ }^{24}$ M. E. Rose, Elementary Theory of Angular Momentum (Wiley, New York, 1957).

${ }^{25}$ A. R. Edmonds, Angular Momentum in Quantum Mechanics (Princeton University Press, Princeton, 1957).

${ }^{26} \mathrm{~J}$. F. Reading, Phys. Rev. A $\underline{8}, 3262$ (1973).

${ }^{27}$ A. L. Ford, J. F. Reading, and R. L. Becker, J. Phys. B $\underline{12}, 2905$ (1979). 
${ }^{28}$ J. F. Reading and A. L. Ford, Phys. Rev. A $\underline{21}, 124$ (1980).

${ }^{29} \mathrm{~J}$. Kirsch, B. Müller, and W. Greiner, Z. Naturforsch. 35A, 579 (1980).

${ }^{30} \mathrm{G}$. Wunner, Phys. Rev. Lett. $\underline{42}, 79$ (1979).

${ }^{31}$ C. S. Warke, Physics News 8,1 (1977).

${ }^{32}$ R. K. Bhaduri, Y. Nogami, and C. S. Warke, Astrophys. J. 217, 324 (1977).

${ }^{33}$ C. S. Warke and A. K. Dutta, Phys. Rev. A 16,1747 (1977).

${ }^{34} \mathrm{P}$. Achuthan, T. Chandramohan, and K. Venkatesan, J. Phys. A 12, 2521 (1979).

${ }^{35}$ M. Shinada, Phys. Lett. 74A, 401 (1979).
${ }^{36}$ V. N. Oraevskii, A. I. Rez, and V. B. Semikoz, Zh. Eksp. Teor. Fiz. 72, 820 (1977) [Sov. Phys. JETP $\underline{45}$, 428 (1977)].

${ }^{37}$ V. N. Oraevskii, A. I. Rez, and V. B. Semikoz, Yad. Fiz. 28, 1123 (1978) [Sov. J. Nucl. Phys. 28, 577 (1978)].

${ }^{38} \mathrm{~T}$. K. Dmitrieva and G. I. Plindov, Phys. Lett. 71A. 326 (1979).

${ }^{39}$ K. A. U. Lindgren and J. T. Virtamo, J. Phys. B $\underline{12}$, 3465 (1979).

${ }^{40} \mathrm{~J}$. T. Virtamo and K. A. U. Lindgren, Phys. Lett. $71 \mathrm{~A}$, 329 (1979).

${ }^{41} \mathrm{~J}$. Simola and J. Virtamo, J. Phys. B $\underline{11} 3309$ (1978). 